\title{
Exceptions to recognition failure as a function of the encoded association between cue and target
}

\author{
DAVID J. BRYANT \\ Stanford University, Stanford, California
}

\begin{abstract}
The relation of the recognition failure of recallable words to overall recognition rates is largely invariant across conditions that influence both recall and recognition separately. In two experiments, the influence of the integration of the members of A-B word pairs on this relation was investigated. In Experiment 1, it was found that deviations of observed recognition failure from predictions of the Tulving-Wiseman function (Tulving \& Wiseman, 1975) were produced by shallow, nonsemantic encoding. In Experiment 2, the association of category-to-instance pairs was varied. It was found that weak associates caused larger deviations of observed recognition failure from predicted recognition failure than did strong associates. Such results suggest that a strongly encoded association between cue and target elements of A-B pairs is a necessary condition for the adherence of data to the Tulving-Wiseman function. The implications of these findings for general models of memory are discussed.
\end{abstract}

Recognition failure of recallable words, or, simply, recognition failure, is found in experiments in which subjects study A-B word pairs and complete successive recognition and cued recall tests. Recognition failure is the phenomenon that a proportion of words recalled on the cued recall test are not recognizable by the same subject, and it is typically measured by the conditional probability of recognition given recall, $P(R n \mid R c)$, where $R n$ stands for recognition hits and $R c$ for cued recall. ${ }^{1}$ An important finding has been that the magnitude of recognition failure is predictable on the basis of overall recognition rates by a mathematical function, the Tulving-Wiseman function (Tulving \& Wiseman, 1975), which has the following form:

$$
P(R n \mid R c)=P(R n)+.5\left[P(R n)-P(R n)^{2}\right] .
$$

Recognition failure data adhere closely to this function across diverse experimental situations, as, for example, when the to-be-remembered items have one or many meanings (Tulving \& Watkins, 1977), when the cue words

The research reported in Experiment 1 was conducted at the University of Toronto and was supported by a grant from the Natural Sciences and Engineering Research Council of Canada (No. A8632) to Endel Tulving. The research reported in Experiment 2 was conducted at Stanford University and was supported by National Institute of Mental Health Grant MH 13950 and AFOSR Grant 87-0282 to Gordon H. Bower. I am extremely grateful to Endel Tulving and Gordon Bower for their guidance, support, and invaluable advice. Thanks also go to Karen Murphy for her assistance with Experiment 2 and to Chris Kortge, Frank Bellezza, John Gardiner, Lars-Göran Nilsson, and Jeroen Raaijmakers for their helpful comments. Correspondence concerning this article should be addressed to David Bryant, Department of Psychology, Stanford University, Stanford, CA 94305. Electronic mail may be sent to bryant@psych.stanford.edu. are nouns or adjectives (Bartling \& Thompson, 1977), when the retention intervals vary from a few minutes (see, e.g., Begg, 1979) to 4 weeks (Donnelly, 1988), when recall is greater than recognition (Wiseman \& Tulving, 1976), or when recognition is greater than recall (see, e.g., Postman, 1975). Thus, many manipulations that affect recall and recognition separately do not influence the rate of recognition failure or its relation to overall recognition rates. In fact, the regularity of the relation between recognition failure and recognition rates has led to proposals that the Tulving-Wiseman function has the status of an empirical law (Gardiner \& Nilsson, 1990; Jones, 1983, 1984; Nilsson, Dinniwell, \& Tulving, 1987; Nilsson, Law, \& Tulving, 1988).

The regularity of recognition failure data makes recognition failure an important phenomenon for theories of human memory. The Tulving-Wiseman function establishes a constraint on the relation between recall and recognition that must be satisfied by any general model of memory. Recognition and recall are largely independent; when the conditional probability of recognition, given recall, is plotted against the overall probability of recognition, data points tend to lie near the diagonal, indicating stochastic independence. The constant $c$ in the TulvingWiseman function is an index of dependence between recognition and recall (Tulving \& Wiseman, 1975). Complete independence would be represented by $c=0$; complete dependence, by larger values of $c$ (the upper boundary of $c$ varies inversely with the recognition rate). Although the observed value, $c=.5$, indicates a fairly large degree of independence, there is a small but reliable degree of dependence evident between recognition and cued recall. Any model intended to account for both recognition and cued recall should be able to explain the Tulving-Wiseman 
function and why recognition and recall are largely, but not perfectly, independent. A number of models do, in fact, offer explanations of the occurrence and magnitude of recognition failure in some fashion (for a review, see Ratcliff \& McKoon, 1989). Hintzman (1987), for example, has directly applied his MINERVA 2 model to the recognition failure paradigm and successfully reproduced the major findings.

Despite the generality of the Tulving-Wiseman function, in several studies levels of recognition failure have been reported that were much lower than what the TulvingWiseman function would predict. Such deviations of observed recognition failure from predicted recognition failure can be termed exceptions to recognition failure. These exceptions have been observed when the A-B study pairs consisted of abstract words or number-word pairs (Tulving \& Watkins, cited in Gardiner \& Tulving, 1980), weakly related words (Neely \& Payne, 1983), or unique famous names (Muter, 1984). Another important finding is that little recognition failure is evident when word pairs are studied rotely (Begg, 1979). In addition, A-B pairs consisting of a conceptual category paired with an instance of that category can produce deviations from the TulvingWiseman function (Nilsson \& Shaps, 1980, 1981). On the basis of these findings, Nilsson et al. (1988) have argued that there are two classes of exceptions-those produced by poor integration of cue and target, and those produced by informational overlap in recall and recognition cues (see also Gardiner, 1989; Gardiner \& Nilsson, 1990). Such exceptions to recognition failure are important for several reasons. First, they establish the boundaries of the Tulving-Wiseman function as an empirical law (Nilsson et al., 1988). That is, they define the conditions under which the function accurately predicts recognition failure. Second, if exceptions to recognition failure are themselves regular and predictable, they form further constraints on memory models. Given the number of models that simulate recognition and recall, one task of memory researchers should be to model the relationship between the two. By understanding why certain situations produce exceptions to recognition failure, one may be able to distinguish theoretical accounts of memory processes.

To use exceptions to recognition failure as constraints on theory, however, we need a better understanding of the factors associated with such exceptions. Tulving and associates have, at various times, proposed that exceptions to recognition failure occur because of inadequate encoding of the A-B pairs (Gardiner \& Tulving, 1980) or weak association of the A and B items (Nilsson et al., 1988). These explanations are closely related, since poor encoding of word pairs presumably results in weak associations. This general explanation is supported by findings that elaborative encoding instructions can significantly increase the observed recognition failure for abstract wordword and number-word pairs, which normally show little recognition failure (Gardiner \& Tulving, 1980).

However, the specific claims that type of encoding and degree of association determine the level of recognition failure have not been fully tested. Gardiner and Tulving (1980) found increased recognition failure for unrelated pairs with highly elaborative study instructions, but they did not examine the effect of nonintegrative encoding tasks. Fisher (1979) found that repetition study instructions produced exceptions to recognition failure only for unrelated pairs, not for pairs with a preexisting association. To date, Begg's (1979) exception resulting from rote study of materials remains the best demonstration that type of encoding influences the amount of observed recognition failure. The present study consists of an attempt to test the validity of the first class of exceptions proposed by Nilsson et al. (1988), those due to encoding manipulations. In the experiments that follow, the effects of level of processing (Craik \& Lockhart, 1972; Craik \& Tulving, 1975 ) and associability of cue and target items on recognition failure were examined.

\section{EXPERIMENT 1}

Experiment 1 was carried out to determine whether shallow, nonsemantic encoding of word pairs produces exceptions to recognition failure. This study relies on the levels-of-processing framework (Craik \& Lockhart, 1972; Lockhart, Craik, \& Jacoby, 1976), within which memory is related to the semantic quality of encoding, such that more elaborative semantic encoding generally produces better recall and recognition (Craik \& Tulving, 1975). There is also evidence that shallow levels of processing produce less integration of target and context information. For example, unique retrieval cues provide less facilitation to recall at lower levels of processing (Moscovitch \& Craik, 1976), and incongruence of cue and target words has a more detrimental effect on recall, given semantic as opposed to phonetic encoding (Fisher \& Craik, 1977).

In Experiment 1, the standard levels-of-processing paradigm (e.g., Craik \& Tulving, 1975) was combined with the standard recognition failure paradigm (Tulving, 1983, p. 274). The subjects studied word pairs by answering questions about the structural, phonetic, or semantic properties of each pair, and they then completed a recognition and cued recall test. On the basis of the views of Gardiner and Tulving (1980) and Nilsson et al. (1988), it was expected that recognition failure would deviate from predictions of the Tulving-Wiseman function in the structural and phonetic encoding conditions, but not in the semantic condition.

\section{Method}

Subjects. Twenty-four University of Toronto undergraduates participated in exchange for credit in an introductory psychology class. The subjects were run in groups of 1 to 6 .

Materials. A pool of 72 common words was selected from pools used by Craik and Tulving (1975) and Watkins and Tulving (1975) and from the New Roget's Thesaurus (1959). This critical pool was divided into 36 cue words and 36 target words, which were paired on the basis of rhyme or conceptual relatedness or both. Some examples of word pairs used in Experiment 1 are shown in Table 1. Four patterns of upper- and lowercase lettering were used, as is 
Table 1

Example of Cue-Target Pairs in Experiment 1

\begin{tabular}{ll}
\hline \multicolumn{1}{c}{ Pair } & \multicolumn{1}{c}{ Classification } \\
\hline LUTE-flute & rhyming/related \\
mast-rudder & nonrhyming/related \\
crate-STATE & rhyming/unrelated \\
HOPE-TENNIS & nonrhyming/unrelated \\
\hline
\end{tabular}

also shown in Table 1. Critical pairs were divided into three sets of 12 , each containing 3 examples of the four types of pairs and four patterns of printing.

A pool of 108 noncritical cue and target words was selected from the same sources as were the critical materials. Noncritical materials were paired in the same fashion as critical materials were, except that no pairs contained words that both rhymed and were conceptually related. The filler pairs were divided into two sets of 54 , the beginning and ending sets, which preceded and followed the critical items during presentation. Two sets of 18 practice word pairs were constructed in the same way as the filler materials were. The critical, noncritical, and practice items were printed on $4 \times 6$ in. index cards for presentation.

The recognition test consisted of 144 single words printed on two sheets of paper, 36 of which were the critical target words randomly scattered amid 108 previously unseen distractor items. None of the filler items appeared on either the recognition or the recall test. Critical words appeared in the letter case that they had appeared in during study, and a total of 72 test items appeared in each letter case. Test items appeared in three columns on each sheet with the words "Yes" and "No" printed beside each word. The cued recall test consisted of the 36 critical cue words printed in two columns on a single sheet of paper.

Design. The independent variables were the type of study question used to encode a given word pair, with three levels (structural, phonetic, and semantic), and response type, with two levels (positive or negative). The dependent variables were the number of previously studied target words correctly indicated as having occurred during study (recognition) and the number of previously studied target items produced in response to the appropriate cue word (recall). The design was a within-subjects randomized design.

Each set of critical pairs was studied according to a different level of study question by individual subjects. An equal number of subjects studied each set at each level of study question. This procedure, combined with the design of the materials, ensured that every critical item was studied an equal number of times at each level of study question, that each subject answered an equal number of structural, phonetic, and semantic questions, and that, for each question type, equal numbers of positive and negative responses were required. The order of presentation was randomly determined, except that the beginning filler set preceded, and the end set followed, the critical pairs.

Procedure. The subjects were told that they were participating in an experiment on human memory and that they would study word pairs and complete two memory tests for the second word of each pair. Before the experimental trial, the subjects were familiarized with the study and test procedures by completing two 18 -item practice trials.

The subjects studied each word pair by answering a verbally presented study question. Three questions were used: (1) "Are the words printed in the same lettering case?" (structural); (2) "Do the words rhyme?" (phonetic); and (3) "Are the words meaningfully related?" (semantic). It was explained that the semantic question required a judgment of the conceptual or categorical relationship between the pair members. The pairs were displayed visually for approximately $2 \mathrm{sec}$. The subjects recorded their responses to study questions by circling either "Yes" or "No" for each item on an answer sheet provided.

After a 5-10 min interval, during which they worked on an unrelated task, the subjects completed the recognition test. On the recognition test, they indicated the words that they believed had appeared during study by circling the "Yes" next to each, and those they believed had not appeared by circling the "No." The test was self-paced. Immediately after the recognition test, the subjects completed the cued recall test. They printed as many of the target words as possible next to the appropriate cue word. This test was also self-paced.

\section{Results}

Recognition was defined as circling the "Yes" next to a previously studied target word; recall, as the production of a previously studied target word in response to the cue word it had been paired with at study. Items were independently classifiable on the basis of level of study question and response type (positive/negative). The data for each condition, collapsed across subjects and items, are presented in Table 2, along with the fourfold classification of items in terms of recognition and recall success and failure. The data, collapsed across response type, are also displayed graphically in Figure 1. The first two columns of Table 2 represent the overall recognition and recall rates. Columns 3-6 contain the $2 \times 2$ recognition/ recall classification. The next two columns indicate the value of $P(R n \mid R c)$ predicted by the Tulving-Wiseman function and that observed in the data. The next column indicates the absolute deviation of observed from predicted

Table 2

Proportion of Items Recognized and Recalled, and a $2 \times 2$ Classification of Recognition and Recall with Predicted and Observed $P(R n \mid R c)$ Values and Observed Deviations from the Tulving-Wiseman Function (Experiment 1)

\begin{tabular}{|c|c|c|c|c|c|c|c|c|c|c|}
\hline \multirow[b]{2}{*}{ Condition } & \multirow[b]{2}{*}{$R n$} & \multirow[b]{2}{*}{$R c$} & \multicolumn{2}{|c|}{$R n$} & \multicolumn{2}{|c|}{$\bar{R} n$} & \multicolumn{2}{|c|}{$P(R n \mid R c)$} & \multirow[b]{2}{*}{ Deviation } & \multirow[b]{2}{*}{$P(R c \mid R n)$} \\
\hline & & & $R c$ & $\overline{R c}$ & $R c$ & $\overline{R c}$ & Predicted & Observed & & \\
\hline Structural & .56 & .28 & .24 & .32 & .04 & .40 & .68 & .85 & .17 & .43 \\
\hline Positive & .58 & .26 & .23 & .35 & .03 & .39 & .70 & .89 & .19 & .39 \\
\hline Negative & .54 & .31 & .25 & .29 & .05 & .40 & .66 & .82 & .16 & .46 \\
\hline Phonetic & .69 & .38 & .35 & .34 & .03 & .28 & 80 & .93 & .13 & .51 \\
\hline Positive & .69 & .41 & .38 & .31 & .03 & .28 & .80 & .93 & .13 & .55 \\
\hline Negative & .69 & .35 & .33 & .37 & .03 & .28 & .80 & .92 & .12 & .47 \\
\hline Semantic & .84 & .55 & .50 & .33 & .04 & .12 & .91 & .92 & .01 & .60 \\
\hline Positive & .89 & .77 & .72 & .19 & .05 & .04 & .94 & .93 & -.01 & .79 \\
\hline Negative & .77 & .33 & .30 & .47 & .03 & .19 & .86 & .90 & .04 & .39 \\
\hline
\end{tabular}

Note-False-alarm rate $=.24$. 




Figure 1. Proportion of recallable words recognized, as a function of overall recognition rate, in Experiment 1 . The curved solid line represents the Tulving-Wiseman function; the straight dashed line, the stochastic independence of recall and recognition.

$P(R n \mid R c)$ values. The final column contains the conditional probability of recalling a word that was recognized, $P(R c \mid R n)$.

Both recognition and recall increased as a function of depth of processing. A complement of statistical analyses were performed on the overall recognition and recall data. The results were in line with those reported by Craik and Tulving (1975), except that no significant effect of response type was found for recognition.

Of primary interest was the deviation in each condition of $P(R n \mid R c)$ from the value predicted by the TulvingWiseman function. As can be seen in Table 2, the absolute deviation of observed from predicted values of $P(R n \mid R c)$ varied with type of study question, with greater deviation evident at lower levels of processing. Deviation scores for individual subjects were submitted to an analysis of variance, which revealed a significant effect of study question $[F(2,46)=6.43, p<.01]$ but not of response type $[F(1,23)=0.389, p>.05]$. The interaction of response type and study question was also not significant $[F(2,46)=0.617, p>.05]$.

In the semantic condition, positive response items were semantically related, whereas negative response items were unrelated. The lack of a significant difference between these items indicates that the semantic relatedness of pairs did not influence recognition failure when subjects made a semantic judgment. Although this analysis does not indicate whether semantic relatedness affected recognition failure in the phonetic or structural conditions, it is unlikely that it would, considering that subjects were making rhyme or letter-case judgments and presumably not paying much attention to the meaning of the words.
The level of recognition failure in each condition was determined by the nature of the encoding task and not the materials.

A binomial test used by Neely and Payne (1983) was employed to evaluate the significance of the deviation of observed from predicted recognition failure. This test used the normal approximation to the binomial distribution with $z=($ Obs. - Pred. $) / \sqrt{\text { Pred. } .(1-\text { Pred } .)} / N$, where Obs. is the observed $P(R n \mid R c)$, Pred. is the predicted $P(R n \mid R c)$, and $N$ is the number of words recalled. The $z$ value was calculated for each condition; within all three conditions, the results for positive and negative responses were the same as the result for the overall condition. The observed $P(R n \mid R c)$ was not significantly different from the predicted value in the semantic condition $(z=0.44, p>.05$, two-tailed), but it was significantly greater in both the phonetic $(z=3.39, p<.01$, two-tailed) and the structural ( $z=3.28, p<.01$, two-tailed) conditions. Thus, observed recognition failure conformed to the TulvingWiseman function only in the semantic condition, and it deviated significantly from prediction given phonetic or structural encoding. The deviation was somewhat larger in the structural than in the phonetic condition, but this difference was not statistically significant.

Cued recall performance decreased with nonsemantic encoding, implying low integration of cue and target items. To verify this conclusion further, the conditional probability of recall given recognition, $P(R c \mid R n)$, was calculated for each condition (see Table 2 ). This measure indicates the recallability of recognizable items, or the effectiveness of context cues in cuing recall, independent of the recognizability of the target item; and higher levels of $P(R c \mid R n)$ indicate greater integration of the cue and target words. The overall value of $P(R c \mid R n)$ increased with depth of processing. An analysis of variance revealed significant effects of study question $[F(2,46)=8.00$, $p<.01]$ and response type $[F(1,23)=12.30, p<.01]$, as well as a significant interaction between them $[F(2,46)$ $=10.20, p<.01]$. These results indicate that, overall, study question was an effective manipulation of integration of cue and target words and that the structural and phonetic conditions produced relatively low levels of integration. However, $P(R c \mid R n)$ of negative response items did not increase with depth of processing. The interaction of study question and response type seems to reflect this finding as well as the relatively large effect on $P(R c \mid R n)$ of response type in the semantic condition. The degree of integration was higher for positive rather than negative response items, except in the structural condition.

\section{Discussion}

The results of this experiment demonstrate that shallow, nonsemantic encoding produces larger values of $P(R n \mid R c)$ than the Tulving-Wiseman function predicts. Note that the same word pairs were used in all three conditions, and that unlike what has been found in some previous studies (e.g., Gardiner \& Tulving, 1980; Muter, 1984), the exceptions to recognition failure found in the 
structural and phonetic conditions occurred despite the use of materials sufficient for normal levels of recognition failure in the semantic condition. Semantic encoding was associated with a greater conditional probability of recall given recognition than nonsemantic encoding, indicating greater integration of cue and target words in the semantic condition. One interpretation of the present findings is that nonsemantic encoding resulted in exceptions to recognition failure because such encoding did not sufficiently associate the A and B items in memory (see Nilsson et al., 1988). To explore this possibility further, the preexisting strength of association between categorized cue and target items was varied in Experiment 2.

\section{EXPERIMENT 2}

The purpose in Experiment 2 was to determine whether exceptions to recognition failure could be found within the semantic level of processing when the strength of association between cue and target items was varied. Associative strength has been manipulated in a number of previous studies (e.g., Neely \& Payne, 1983; Vining \& Nelson, 1979; Watkins \& Tulving, 1975; Wiseman \& Tulving, 1976), with mixed results. Watkins and Tulving (1975) and Wiseman and Tulving (1976) reported that recognition failure conforms to the Tulving-Wiseman function when weakly associated or unrelated word pairs are used. Vining and Nelson (1979) also found that recognition failure with weakly related word pairs conformed to the function, although their strongly related items produced more recognition failure than had been predicted. On the other hand, Fisher (1979) obtained an exception to recognition failure for weakly related pairs when subjects studied the pairs by repetition. Neely and Payne's (1983) weakly related pairs likewise produced higher conditional probabilities of recognition, given recall, than had been predicted. They hypothesized that this effect was due to some change in encoding strategy brought on by their having had the subjects study the items in the same list with strongly related name pairs. In fact, in the studies in which predicted levels of recognition failure for weakly related pairs were reported, study-to-learn instructions were used, and the subjects were free to search for, or devise, some relation between the nominally unrelated items (cf. Bryant, 1990). In the current study, the subjects' study task was to make category membership judgments. This was expected to focus subjects' attention on the preexisting relationship between cue and target and induce subjects to encode the items in terms of that relationship.

The subjects studied category-instance pairs by making a category judgment about each pair. The instances were strong associates, weak associates, or nonassociates of the category with which they were paired. There have been reports that categorized materials produce low levels of recognition failure (Nilsson \& Shaps, 1980, 1981; Park \& Auble, cited in Tulving, 1983, p. 289), which has been attributed to informational overlap of cue and target (see Gardiner \& Nilsson, 1990; Nilsson et al., 1987). Nilsson and Shaps (1981) found no difference in the degree of deviation of observed from predicted recognition failure between strong and weak category associates. However, their study was hampered by high overall recognition rates (on the order of .87 to .93 ), which may have restricted the amount of recognition failure possible and masked a difference between the conditions. In Experiment 2, the attempt was made to determine whether weak category associates exhibit greater deviation from the TulvingWiseman function than strong associates when recognition rates are lower. To reduce the use of category information during recognition and ensure the potential for normal degrees of recognition failure, each category in the present experiment was unique, and distractor items on the recognition test were drawn from the same categories as target items. It was expected that nonassociates and weak associates of a category would not be well integrated with their category cues and would therefore show greater deviation in recognition failure from the Tulving-Wiseman function than strong associates.

\section{Method}

Subjects. Thirty Stanford University undergraduates participated either for credit in an introductory psychology class or for pay. The subjects were run individually.

Materials. To form the critical materials, a pool of 36 categories was drawn from the norms of Battig and Montague (1969). A strong and weak associate of each category was selected. The strong associates had a mean frequency of generation in response to their category of 355.75; the weak associates, a mean frequency of 1.42. In addition, 36 nouns unrelated to any category were selected from the materials used in Experiment 1. Examples of the categories and target words are shown in Table 3 . A pool of 48 filler categoryword pairs was constructed. Sixteen of the words were strong associates, 16 weak associates, and 16 nonassociates to their category. This pool was divided into 24 precritical and 24 postcritical pairs.

The recognition test consisted of 144 single words, 36 of which were the critical target words scattered amid 108 previously unseen distractor items. The test items were printed in alphabetical order in two columns on three sheets of paper. Beside each test item was a scale from -3 to +3 (with no " 0 "), which subjects used to make their recognition and confidence judgments. The distractor items were a strong associate, weak associate, and nonassociate selected from Battig and Montague (1969) for each critical category. The cued recall test consisted of the 36 critical categories printed in two columns on a single sheet.

Design. The independent variable was the strength of association between the target word and its category cue (strength of association) with three levels (nonassociate, weak associate, and strong associate). As in Experiment 1, dependent variables were recognition and cued recall, and the design was a within-subjects randomized design. Each critical category had a strong associate, weak

Table 3

Example of Category-Word Pairs in Experiment 2

\begin{tabular}{llll}
\hline & \multicolumn{3}{c}{ Strength of Association } \\
\cline { 2 - 3 } \multicolumn{1}{c}{ Category } & Strong & Weak & Non \\
\hline an occupation & doctor & actress & column \\
a vegetable & carrot & pumpkin & market \\
a fish & trout & anchovy & subject \\
a unit of time & hour & season & planet \\
\hline
\end{tabular}


associate, and nonassociate that could be paired with it, and one third of the subjects studied a category with its strong associate. weak associate, or nonassociate. The order of presentation was randomly determined, except that a filler set preceded and followed the critical items.

Procedure. The subjects were told that they were participating in a memory experiment and that they would study category-word pairs and complete two memory tests for these materials.

The category-word pairs were displayed, one at a time, on a computer screen. The category was displayed on the left side and the target word on the right. The subjects' study task was to indicate whether or not the word was a member of the category by pressing the " $Y$ " key on the computer keyboard for "yes" and the " $N$ " key for "no." Each pair remained on the screen for $2.5 \mathrm{sec}$. After completing the study phase, the subjects worked on an unrelated task for $15 \mathrm{~min}$.

The subjects completed the recognition test first. They indicated whether they believed each test item was a previously studied word by selecting a number from -3 to +3 on the scale beside the word. A negative number meant the word was not a study item; a positive number, that it was. The absolute value of the selection indicated subjects' confidence in their judgment, where " 1 " meant little confidence, " 2 " meant moderate confidence, and " 3 " meant high confidence. The subjects completed the cued recall test immediately after the recognition test, by printing as many of the target words as they could remember next to the appropriate category cue. Both tests were self-paced.

\section{Results}

Recognition was defined as selecting any positive number $(+1$ to +3$)$ for a previously studied target word on the recognition form. Cued recall was defined as the production of a target word in response to its appropriate category cue. The data for each condition, collapsed across subjects and items, are presented in Table 4 , with the $2 \times 2$ recognition/recall classification. The data are also displayed graphically in Figure 2.

Overall recognition rates increased with strength of association $[F(2,87)=5.36, p<.01]$, but recognition of strong and weak associates did not differ significantly $[t(29)=0.58, p>.05]$. Cued recall also increased with strength of association $[F(2,87)=118.87, p<.001]$, and recall of strong associates was significantly greater than that of weak associates $[t(29)=7.41, p<.001]$. This finding indicates that integration of category-instance pairs varied with strength of association and was greatest for strong associates.

The conditional probability of recall given recognition, $P(R c \mid R n)$, was calculated for each condition (see the far right column of Table 4). The value of $P(R c \mid R n)$ increased with strength of association $[F(2,87)=114.65, p<.001]$, and $P(R c \mid R n)$ was greater for strong than for weak asso- ciates $[t(29)=6.94, p<.001]$. This provides further evidence that the experimental manipulation of association strength was successful, even among recognizable items.

Interest centered on the deviation of observed from predicted $P(R n \mid R c)$ values. A binomial test was again applied to evaluate the difference between observed and predicted recognition failure. The observed $P(R n \mid R c)$ was significantly greater than the predicted value in all three conditions: strong associates $(z=3.10, p<.01)$, weak associates $(z=5.70, p<.01)$, and nonassociates $(z=$ $2.95, p<.01)$. Thus, as can be seen in Figure 2 , $P(R n \mid R c)$ deviated from prediction to some degree in all conditions. However, the degree of deviation decreased with strength of association $[F(2,87)=17.47, p<.001]$, and deviation was much greater for weak associates than for strong associates $[t(29)=3.07, p<.01]$. The degree of deviation was roughly the same for weak associates and nonassociates, and much greater in these two conditions than in the strong associate condition.

\section{Discussion}

The results of this experiment demonstrate that weak associations between the cue and target elements of a pair produce larger values of $P(R n \mid R c)$ than the TulvingWiseman function predicts, as well as greater dependency of recall and recognition. Although observed recognition failure did not conform closely to the Tulving-Wiseman function in the strong associate condition, the deviation in this condition was considerably less than it was for weak associates. This result supports the findings of Fisher (1979) and Neely and Payne (1983) that weakly related pairs produce exceptions to recognition failure. Unlike in Fisher's (1979) study, however, in the present experiment the subjects engaged in a semantic study task. The result contradicts earlier findings that recognition failure of unrelated pairs conforms to the Tulving-Wiseman function (Watkins \& Tulving, 1975; Wiseman \& Tulving, 1976). Presumably, in these studies, subjects were able to sufficiently associate the paired items during study, despite their lack of strong preexisting associations. There is a growing body of evidence that items that are not integrated at study exhibit less recognition failure than would be predicted (Experiment 1; Begg, 1979; Gardiner \& Tulving, 1980). The category judgment task of this experiment seems to have focused subjects on the preexisting association during encoding, allowing strength of association to affect recognition failure.

The deviation of recognition failure from prediction in the strong condition replicates Nilsson and Shaps's (1980,

Table 4

Proportion of Items Recognized and Recalled, and a $2 \times 2$ Classification of Recognition and Recall with Predicted and Observed $P(R n \mid R c)$ Values and Observed Deviations from the Tulving-Wiseman Function (Experiment 2)

\begin{tabular}{|c|c|c|c|c|c|c|c|c|c|c|}
\hline \multirow[b]{2}{*}{ Strength } & \multirow[b]{2}{*}{$R n$} & \multirow[b]{2}{*}{$R c$} & \multicolumn{2}{|c|}{$R n$} & \multicolumn{2}{|c|}{$\overline{R_{n}}$} & \multicolumn{2}{|c|}{$P(R n \mid R c)$} & \multirow[b]{2}{*}{ Deviation } & \multirow[b]{2}{*}{$P(R c \mid R n)$} \\
\hline & & & $R$ & $\overline{R c}$ & $R c$ & $\overline{R C}$ & Predicted & Observed & & \\
\hline Non & .53 & .06 & .06 & .48 & .003 & .46 & .65 & .95 & .30 & .11 \\
\hline Weak & 63 & .35 & .34 & .30 & .01 & .35 & .75 & .97 & .22 & .53 \\
\hline Strong & .65 & .60 & .51 & .14 & .09 & .26 & .76 & 85 & .09 & .78 \\
\hline
\end{tabular}

Note-False-alarm rate $=.14$ (strong associate distractors), .09 (weak associate distractors), .20 (nonassociate distractors). 


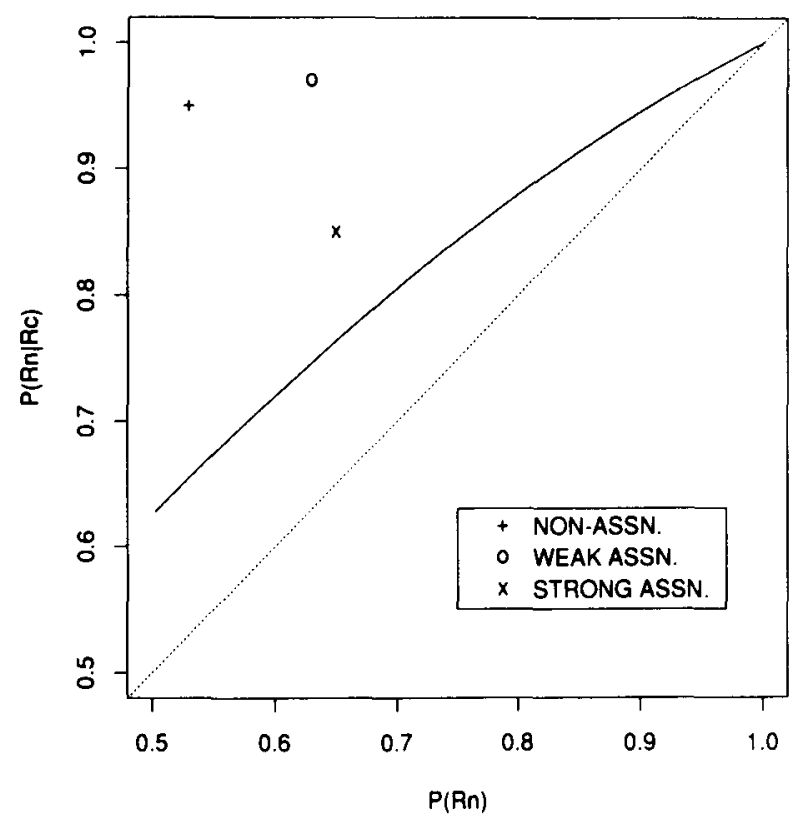

Figure 2. Proportion of recallable words recognized, as a function of overall recognition rate, in Experiment 2.

1981) findings that categorized lists produce exceptions to recognition failure. Why did recognition failure deviate from the Tulving-Wiseman function when the target and cue were strongly associated? When attempting to recognize categorized materials, subjects can use reconstructive retrieval strategies. Subjects in this experiment may have attempted to use their knowledge of the categories of strong associates to aid their recognition of target words. This would essentially turn the recognition test into a cued recognition test and introduce a degree of correlation between recognition and recall because of informational overlap in recall and recognition cues, diminishing the amount of observed recognition failure.

Nilsson and Shaps (1981), however, reported no difference in the deviation of observed recognition failure from predicted levels between strong and weak associates, although a large difference was found in the present study. There are two possible explanations. First, recognition rates were much lower in this experiment, and there was a greater range for weak and strong associates to differ in $P(R n \mid R c)$. Second, extremely weak associates were selected as target items for the weak associate condition in this experiment. Associative strength frequently has only a small effect on measures of memory (Kausler, 1974, pp. 99, 155). Thus, Nilsson and Shaps's (1981) failure to find greater deviation in recognition failure in their weak associate condition may reflect the use of insufficiently weak category instances.

\section{GENERAL DISCUSSION}

The results of two experiments support the general explanation of exceptions to recognition failure put forward by Gardiner and Tulving (1980) and Nilsson et al. (1988).
Poor encoding of the to-be-remembered items-specifically, the lack of strong association between the elements of the word pairs-resulted in levels of recognition failure that deviated from the Tulving-Wiseman function. In Experiment 1 , it was demonstrated that shallow, nonsemantic encoding tasks produce less integrated representations of items and less recognition failure than is predicted. In Experiment 2, it was demonstrated that when the target word of category-instance pairs is an extremely weak associate of its category, recognition failure deviates from the Tulving-Wiseman function to a greater extent than when the target item is a strong associate. In both experiments, the conditions that produced the largest deviations of observed recognition failure from predicted recognition failure were associated with the lowest conditional probabilities of recall given recognition. The results serve to indicate that when the integration of the A-B pair is low, atypical recognition failure data is obtained. Moreover, the deviations were all in the direction of fewer instances of recognition failure. Thus, shallow encoding and weak association of items in A-B pairs lead to greater positive correlation or dependency between recall and recognition.

Why do weak associations between cue and target items produce greater dependence of recall and recognition? One possible explanation involves the unitary trace assumption contained in Flexser and Tulving's $(1978,1982)$ retrieval independence model-the assumption that cue and target items are stored in a single trace or integrated unit in memory. The results of the present experiments support the contention that an integrated representation of the A-B word pair is a necessary condition for the occurrence of recognition failure and the independence of recognition and recall. Shallow encoding that failed to associate the cue and target items would place recognition failure data outside the predictive realm of the TulvingWiseman function.

Flexser and Tulving's $(1978,1982)$ model also predicts that the greater the informational overlap of recall and recognition cues (represented in the model by parameter $k$ ), the greater will be the dependence between the two measures. If cue and target items are not well integrated in memory, the cue word will be ineffective as a retrieval cue on the recall test. In this case, successful recall would depend to a greater extent on the subject's ability to generate the target item from context information common to both cue and target or from information pertaining to the target item itself (as in free recall). Within the framework of the model, weak integration of pairs restricts the set of features of the cue item that are effective for retrieval of the target item. In other words, the set of effective cues to the target is biased toward features of the target, or features shared by the cue and target items. This establishes a degree of informational overlap in the effective recall and recognition cues employable by the subject, a situation that falls under the second class of exceptions to recognition failure described by Nilsson et al. (1988).

Another possible explanation comes from Begg's (1979) "Vandal" theory. This theory states that if a proportion of memory traces of encoded pairs are lost, either in an 
all-or-none fashion or feature by feature, both recognition and cued recall of the pairs will be affected. This would result in a correlation between the two processes. Thus, any factor that promotes greater trace loss will increase the dependence of recall and recognition, and one would expect an exception to recognition failure. Begg (1979) supported his claim with the finding that rote study, which should promote greater trace loss than meaningful study, produces almost no recognition failure. The shallow encoding conditions of Experiment 1 in the present study might also have promoted a high degree of trace loss, accounting for the relatively high levels of $P(R n \mid R c)$ observed in those conditions. The findings in Experiment 2, however, pose a problem for this account. Begg (1979) claims that the integration of items in a pair does not influence recognition failure, provided that the encoding of the pair elements is equivalent. Thus, separate imagery study instructions produced as much recognition failure as did interactive imagery instructions (Begg, 1979). However, in Experiment 2, weak associates, although recognized as frequently as strong associates, displayed virtually no recognition failure. It seems that the integration of paired elements does play a role in recognition failure, even when items are studied according to the same instructions.

\section{THE GENERALITY OF THE TULVING - WISEMAN FUNCTION}

Recognition failure is a general phenomenon that occurs in diverse experimental situations. More importantly, the magnitude of recognition failure is predictable by the Tulving-Wiseman function across these various conditions (see Tulving, 1983). Nilsson et al. (1987) and Nilsson et al. (1988) have reported experiments in which they sought to define the limits of the Tulving-Wiseman function as an "empirical law." Their aim was to establish a regularity in recognition and recall data that might be fruitfully applied to our theorizing about human memory processes; they demonstrated that normal recognition failure can be found with unique names (Nilsson et al., 1988) and category-instance pairs (Nilsson et al., 1987), even though such materials have previously been associated with exceptions to recognition failure (Muter, 1984; Nilsson \& Shaps, 1980, 1981).

However, exceptions to recognition failure are equally important to the issue of whether or not the TulvingWiseman function represents an empirical law. Like any empirical generalization, the Tulving-Wiseman function does not predict the data in all situations (see Tulving, 1983), and exceptions to recognition failure provide the boundary conditions for the Tulving-Wiseman function. The important finding in the current study is that the situations in which recognition failure deviates from the TulvingWiseman function are systematic. The two experiments reported here support the conclusion that a strong association between the cue and target words of A-B pairs is a necessary condition for the adherence of data to the
Tulving-Wiseman function (cf. Gardiner \& Nilsson, 1990; Nilsson et al., 1988). Any factor that tends to reduce the integration of target and cue words in an A-B pair should produce marked exceptions to recognition failure. This is true of encoding factors such as level of processing as well as material factors such as strength of preexisting association. The results of the current study lend support to the contention that integrated encoding of the elements of the A-B pairs is a boundary condition on the descriptive validity of the Tulving-Wiseman function.

\section{CONSTRAINTS ON MODELS OF MEMORY}

General models of memory should be able to account for recognition, recall, and the relation between the two. Whether one wishes to label the Tulving-Wiseman function an empirical law or not, it is a powerful generalization concerning recognition and recall of word pairs, and several theorists have addressed it in their models of memory (e.g., Anderson, 1983; Gillund \& Shiffrin, 1984; Hintzman, 1986, 1988). Ratcliff and McKoon (1989) offer an excellent review of the explanations of recognition failure offered by several general memory models. I will briefly describe how three of these models deal with the phenomenon.

Anderson's (1983) semantic network model, ACT*, explains the independence of recognition and recall in terms of multiple "senses" of words, represented by separate nodes in long-term memory. Recognition is not correlated with cued recall, because the subject is free to interpret the target word as it appears on a recognition test in a sense different from the sense evoked by the cued recall test. (Note that Nilsson et al., 1988, review evidence for recognition failure with words having a single meaning.) Gillund and Shiffrin's (1984) SAM model (see also Raaijmakers \& Shiffrin, 1981) explains the independence of recall and recognition by means of two factors: (1) Recall is a stochastic process in which some searches can succeed, even though item familiarity is below recognition criterion; and (2) random changes in context are assumed to bias the interpretation of recognition cues, as in ACT* . According to MINERVA 2 (Hintzman, 1987), cue and target words of a pair are stored in a single memory vector, but they have uncorrelated features. Likewise, the features of recognition and recall cues are uncorrelated, and when these cues are compared to memory vectors, recognition and recall success are independent. A slight degree of dependence can be introduced by variability in the goodness of encoding of pairs, which affects both recall and recognition of an item.

These models generally satisfy the constraint of the Tulving-Wiseman function. However, exceptions to recognition failure due to nonintegrative encoding of cuetarget associations offer another constraint for models of recognition and recall. Models should not only account for the typical independence of recognition and recall described by the Tulving-Wiseman function, but should also explain why weak associations between cues and tar- 
gets produce greater dependence of recall and recognition. It should be noted that not just weak preexisting associations cause exceptions to recognition failure. The results of Experiment 1, in which the same materials served in all three conditions, demonstrate that when associations are weakly encoded, there is less recognition failure. Flexser and Tulving's $(1978,1982)$ retrieval independence model, which was designed to explain the Tulving-Wiseman function, can account for the results reported here in terms of a parameter, $k$, governing the amount of informational overlap of cue and target items. The model predicts the current findings by means of the assumption that weak integration of cue and target violates the unitary trace assumption of the model and increases the informational overlap of the recall and recognition cues usable by subjects. However, this model has not been applied beyond recognition failure phenomena, and its generalizability is unclear.

The results of both experiments pose some difficulty for the general models discussed so far. As was noted, $\mathrm{ACT}^{*}$ has difficulty with the phenomenon of recognition failure itself. The assumption in $\mathrm{ACT}^{*}$ that recognition failure only occurs for words with multiple meanings or senses is questionable (Nilsson et al., 1988). Even if this were true, however, it would remain unclear why the encoded association between cue and target would have any bearing on the "senses" assigned to recognition test items. One would have to assume a greater effect of encoding context in the determining of an item's interpretation, given strong cue-to-target associations or semantic encoding. This criticism holds true for SAM also, which invokes context effects during recognition to explain recognition failure. The strength of association between context cues and memory images in SAM could affect the probability that a recall search would succeed for targets that are not recognizable. Weak associations that reduce the probability of such a recall search's succeeding would have the effect of increasing the dependence of recall and recognition. However, Gillund and Shiffrin (1984) stress that, in their view, most recognition failures occur because of context changes that induce different meanings of target items during recognition and recall.

Of the models discussed here, Hintzman's MINERVA 2 may be the best suited to explain the current results. MINERVA 2 is conceptually similar to Flexser and Tulving's (1978) model, which provides a good mathematical description of recognition failure data and could predict the current results. Also, Hintzman (1987) has successfully simulated an exception to recognition failure reported by Muter (1984). Muter (1984) found that subjects exhibited little recognition failure when unique famous names (e.g., Kemal Ataturk, Søren Kierkegaard) were presented as the pairs to be learned, although normal degrees of recognition failure were observed when commonly occurring famous names (e.g., Cooper, Davis) were used as target items. ${ }^{2}$ To model this finding, Hintzman (1987) conjectured that subjects would be unfamiliar with at least some of the unique names and would not encode these items. Thus, in MINERVA 2, Hintzman as- sumed all-or-none encoding of pairs, which introduced a large correlation between recall and recognition, and hence greatly reduced the amount of recognition failure.

The assumption of all-or-none encoding, however, does not capture the manipulation of preexisting or encoded associative strength of paired items. In the experiments reported here, the materials were common words and categories that all subjects should have been familiar with. MINERVA 2 could possibly account for the results with a related assumption that weakly related cue and target words may be encoded in separate memory vectors. In terms of MINERVA 2, one would assume a probability of the features of the cue word being encoded in a vector separate from the vector in which the features of the target are encoded. This probability would reflect cases in which the cue and target were not associated at the time of encoding, depending on the encoding task or on their preexisting association. Weakly associated pairs, or pairs encoded according to shallow, nonintegrative study tasks, would have a higher probability of being encoded separately, making the cue words ineffective retrieval cues for the target words on a recall test. This would in turn introduce a greater dependency between recall and recognition, because (1) recall of the target items encoded in isolation would have to proceed on the basis of item information, as in free recall, ${ }^{3}$ and (2) this would greatly reduce the probability of unrecognizable words' being recalled. Another way of expressing this is to say that the informational overlap of usable retrieval cues on the recall and recognition tests would be greater, resulting in exceptions to recognition failure (Gardiner, 1989; Gardiner \& Nilsson, 1990; Nilsson et al., 1988). The greater the probability of nonassociative encoding, the less recognition failure would occur, and recognition and recall would be largely dependent.

General models of memory have performed fairly well at explaining the occurrence and magnitude of recognition failure. The challenge for these models will be to account for exceptions to recognition failure under conditions leading to weak association of cue and target words. The problem for each of the models described arises from their simple conception of encoding. Although there is growing evidence that associative processes at encoding influence later retrieval (see, e.g., Bryant, 1990; Nelson, McEvoy, \& Schreiber, 1990), these models assume a relatively passive encoding process, relying on retrieval processes to explain recognition failure. The results of the present experiments demonstrate the critical role of encoding in determining the relation between recall and recognition. Truly general models of memory will have to come to terms with encoding factors if they are to continue to expand our insight into human memory.

\section{REFERENCES}

ANDERson, J. R. (1983). The architecture of cognition. Cambridge, MA: Harvard University Press

Bartling, C. A., \& Thompson, C. P. (1977). Encoding specificity: Retrieval asymmetry in the recognition failure paradigm. Journal of Experimental Psychology: Human Learning \& Memory, 3, 690-700. 
Battig, W. F., Montague, W. E. (1969). Category norms of verbal items in 56 categories: A replication and extension of the Connecticut category norms. Joumal of Experimental Psychology Monographs, 80(3, Pt. 2).

BEGG, I. (1979). Trace loss and the recognition failure of recallable words. Memory \& Cognition, 7, 113-123

BryaNT, D. J. (1990). Implicit associative responses influence encoding in memory. Memory \& Cognition, 18, 348-358.

Craik, F. I. M., Lockhart, R. S. (1972). Levels of processing: A framework for memory research. Journal of Verbal Learning \& Verbal Behavior, 11, 671-684.

Craik, F. I. M., \& Tulving, E. (1975). Depth of processing and the retention of words in episodic memory. Joumal of Experimental Psychology: General, 104, 268-294.

Donnelly, R. E. (1988). Priming effects in successive episodic tests. Journal of Experimental Psychology: Learning, Memory, \& Cognition, 14, 256-265.

Fisher, R. P. (1979). Retrieval operations in cued recall and recognition. Memory \& Cognition, 7, 224-231.

Fisher, R. P., \& CRAIK, F. I. M. (1977). Interaction between encoding and retrieval operations in cued recall. Journal of Experimental Psychology: Human Learning \& Memory, 3, 701-711.

FleXSER, A. J., \& TUlving, E. (1978). Retrieval independence in recognition and recall. Psychological Review, 85, 153-171

FleXser, A. J., \& Tulving, E. (1982). Priming and recognition failure. Journal of Verbal Learning \& Verbal Behavior, 21, 237-248.

GaRDiner, J. M. (1989). The Tulving-Wiseman law and exceptions to it. In A. F. Bennett \& K. M. McConkey (Eds.), Cognition in individual and social contexts (pp. 253-260). North-Holland: Elsevier.

GARDINER, J. M., NiLsson, L.-G. (1990). Relation between recognition and recall: The Tulving-Wiseman law. Manuscript submitted for publication.

Gardiner, J. M., \& TUlving, E. (1980). Exceptions to recognition failure of recallable words. Journal of Verbal Learning \& Verbal Behavior, 19, 194-209.

Gillund, G., \& Shiffrin, R. M. (1984). A retrieval model for both recognition and recall. Psychological Review, 19, 1-65.

Hintzman, D. L. (1986). "Schema abstraction" in a multiple-trace memory model. Psychological Review, 93, 411-428.

Hintzman, D. L. (1987). Recognition and recall in MINERVA 2: Analysis of the 'recognition-failure' paradigm. In P. Morris (Ed.), Modelling cognition (pp. 215-229). New York: Wiley.

HinTZMaN, D. L. (1988). Judgments of frequency and recognition memory in a multiple-trace memory model. Psychological Review, 95, 528-551

JONES, G. V. (1983). Structure of the recall process. Philosophical Transactions of the Royal Society, London, 302B, 373-385.

JoNES, G. V. (1984). Analyzing recognition and recall. Behavioral \& Brain Sciences, 7, 242-243.

KAUSLER, D. N. (1974). The psychology of verbal learning and memory. New York: Academic Press.

Lockhart, R. S., Craik, F. I. M., Jacoby, L. (1976). Depth of processing, recognition and recall. In J. Brown (Ed.), Recall and recognition (pp. 75-102). London: Wiley.

Moscovitch, M., Craik, F. I. M. (1976). Depth of processing, retrieval cues, and uniqueness of encoding as factors in recall. Journal of Verbal Learning \& Verbal Behavior, 15, 447-458.

MUTER, P. (1984). Recognition and recall of words with a single meaning. Journal of Experimental Psychology: Learning, Memory, \& Cognition, 10, 198-202.

NeEly, J. H., PAYNE, D. G. (1983). A direct comparison of recognition failure rates for recallable names in episodic semantic memory tests. Memory \& Cognition, 11, 161-171.
Nelson, D. L., McEvoy, C. L., Schreiber, T. A. (1990). Encoding context and retrieval conditions as determinants of the effects of natural category size. Joumal of Experimental Psychology: Learming, Memory, \& Cognition, 16, 31-41.

New Roget's thesaurus (1959). New York: Putnam.

Nilsson, L.-G., Dinniwell, M. , Tulving, E. (1987). Recognition failure of categorized words. Memory \& Cognition, 15, 389-396.

Nilsson, L.-G., LAW, J., \& Tulving, E. (1988). Recognition failure for recallable unique names: Evidence for an empirical law of memory and learning. Joumal of Experimental Psychology: Learning, Memory, \& Cognition, 14, 266-277

NILSSON, L.-G., \& SHAPS, L. P. (1980). A functional view of memory. In F. Klix \& J. Hoffman (Eds.), Cognition and memory (pp. 4046). Berlin: Deutscher Verlag der Wissenschaften.

Nilsson, L.-G., ShaPS, L. P. (1981). A reconstructive processing interpretation of the recognition failure phenomenon. Acta Psychologica, 47, 25-37.

Postman, L. (1975). Tests of the generality of the principle of encoding specificity. Memory \& Cognition, 3, 663-672.

RaAijmakers, J. G. W., Shiffrin, R. M. (1981). Search of associative memory. Psychological Review, 88, 93-134.

RATCLIF, R, MCKoon, G. (1989). Memory models, text processing, and cue-dependent retrieval. In H. L. Roediger III \& F. I. M. Craik (Eds.), Varieties of memory and consciousness: Essays in honour of Endel Tulving (pp. 73-92). Hillsdale, NJ: Erlbaum.

Tulving, E. (1983). Elements of episodic memory. New York: Oxford University Press.

Tulving, E., \& Watkins, O. C. (1977). Recognition failure of words with a single meaning. Memory \& Cognition, 5, 513-522.

TulviNG, E., \& Wiseman, S. (1975). Relation between recognition and recognition failure of recallable words. Bulletin of the Psychonomic Society, 6, 79-82.

VINING, S. K., \& NELSON, T. O. (1979). Some constraints on the generality and interpretation of the recognition failure of recallable words. American Journal of Psychology, 92, 257-276.

Watkins, M. J. \& Tulving, E. (1975). Episodic memory: When recognition fails. Journal of Experimental Psychology: General, 104, 5-29.

Wiseman, S., \& Tulving, E. (1976). Encoding specificity: Relation between recall superiority and recognition failure. Journal of Experimental Psychology: Human Learning \& Memory, 2, 349-361.

\section{NOTES}

1. A more accurate conceptual measure of recognition failure is $P(\overline{R n} \mid R c)$, the conditional probability that a recalled word is not recognized. The measure $P(R n \mid R c)$, however, has been adopted in numerous other studies (see Tulving \& Wiseman, 1975), and it will be used in this study. It should be noted that $P(R n \mid R c)$ is inversely related to recognition failure; greater values of $P(R n \mid R c)$ indicate less recognition failure.

2. Muter (1984) also gave subjects historical facts as part of the context cue of each pair and had subjects complete the recognition and cued recall tests as semantic memory tests. Nilsson et al. (1988) have reported that when subjects study unique famous names but complete episodic recognition and recall tests, recognition failure conforms to the TulvingWiseman function.

3. This would, of course, require some specification of how free recall proceeds in MINERVA 2.

(Manuscript received June 25, 1990; revision accepted for publication October 11, 1990.) 